\title{
Dynamic network models: introduction to new and interdisciplinary approaches
}

\author{
David Banks
}

Published online: 10 December 2009

(C) Springer Science+Business Media, LLC 2009

On September 26-27, 2005, the National Academies hosted a special conference on dynamic models for networks. The meeting was funded through a research grant from the National Security Agency, with some organizational support from NSF Grant DMS-0437183/SES 0437239. The purpose of the meeting was to bring together researchers in different fields, all of whom were working on aspects of creating mathematical descriptions of network change. This special issue collects a representative but nonrandom sample of the papers presented at that conference.

The most important outcome of the meeting was the cross-fertilization of ideasthere were clear differences between the fields in the methods that were used and how the problems were framed. For example, biologists had developed models for feedback mechanisms, which are not part of the standard toolkit for social network analysts. But the social network analysts had invented latent space models, made more use of covariate information, and were exploring agent-based simulations to permit representation of more complex relationships. Similarly, the neural scientists used point process models to describe spike-train activity in networks, and had specific interest in making inferences about causation. But the telecommunications researchers used heavy-tailed processes to model activity, and focused on network mapping rather than event triggers.

The meeting drew an impressive selection of outstanding researchers. The keynote speakers were John Doyle, from the California Institute of Technology, speaking on "Network Complexity and Robustness"; Eve Marder, of Brandeis, speaking on "The Development and Stability of Biological Networks"; and Steve Borgatti, of Boston College, speaking on "The State of Art in Social Network Analysis". To give a better sense of the intellectual range of the meeting, other prominent speakers included

D. Banks (莐)

Department of Statistical Science, Duke University, Durham, NC 27708, USA

e-mail: banks@stat.duke.edu 
Nancy Kopell, of Boston University, on "Neurons, Networks, and Noise"; Jon Kleinberg, of Cornell, on "Tracking Complex Networks Across Time and Space"; and Ravi Iyengar, at Mount Sinai School of Medicine, on "Information Processing in Cellular Networks". An audio record of the entire meeting and its many other excellent presentations can be obtained by contacting Scott Weidman of the National Academies.

The papers in this special issue were selected somewhat opportunistically from those that were presented, but with an emphasis upon the social network and organizational theory applications that are of central interest to the readers of Computational and Mathematical Organizational Theory. The intent was to present some of the new ideas in network models that are likely to be useful, while avoiding undue emphasis on the nuts and bolts of applications that are specifically related to biological systems or telecommunications infrastructure.

The paper by Peter Hoff represents a survey of several different ways to use latent space procedures in fitting social networks, with illustrations drawn from data on international relations. The paper by Vance, Archie, and Moss applies that kind of latent space model to study social networks of African elephants, resolving a problem concerning the effect of rainy and dry seasons on elephant behavior. Continuing this theme, the paper by Handcock and Morris presents a model for dynamic networks that unifies and generalizes several classical approaches in the area; it is based upon a stochastic process for random mixing of individuals, conditional on node degrees and clustering. As a methodological contrast, the paper by Carley studies social network problems from the standpoint of agent-based models. And the paper by Chen, Bressler, and Ding lays out a new approach to understanding causal mechanisms in signal propagation through networks (exemplified though an application to chimpanzee neural activity). 